Amn. Sci. forest., 1976, 33 (1), 19-26.

\title{
RADIOGRAPHIES DE CAROTTES DE BOIS EN RAYONNEMENT MONOCHROMATIQUE
}

\author{
M. BEDENEAU et A. RIMSKY* \\ Station de Recherches sur la Forêt et l'Environnement. \\ Centre de Recherches forestières d'Orléans, I. N.R.A., \\ Ardon, 45160 Olivet \\ * Laboratoire de Minéralogie, Cristallographie, \\ Université Pierre et Marie-Curie, \\ Laboratoire associé an C.N.R.S., \\ 4 , place Jussieu, \\ 75005 Paris
}

RÉSUMÉ,

Sous l'impulsion de Polge H., l'étude de carottes de bois aux rayons $\mathrm{X}$ a pris récemment un essor considérable.

On substitue ici au rayonnement polychromatique un rayonnement monochromatique, ce qui présente un certain nombre d'avantages.

Les tubes Rx employés en cristallographie utilisent les rayonnements du Cu, du Fe ou Mo, dont on connaît parfaitement les longueurs d'onde et les raies d'émissions.

On peut ainsi calculer avec une grande précision le coefficient d'absorption massique à analyser et la tension à appliquer aux bornes du tube en fonction du résultat choisi. Le contraste est obtenu par variation du temps de pose.

Une autre particularité du rayonnement monochromatique est la cohérence du faisceau, ce qui élimine le problème de l'isodose.

De même, la parfaite connaissance des caractéristiques des films lève l'hypothèque du développement dès lors que tous les grains du film sont développés.

Il est ensuite aisé, contrôlant tous les facteurs de rayonnement, d'absorption et de développement d'analyser les radiographies de carottes de bois ainsi obtenues en vue de quantifier la densité du bois.

\section{INTRODUCTION}

Sous l'impulsion de H. Polge (I966), le développement des prises de radiographie de bois, et de leur exploitation densitométrique, a pris ces dernières années un essor considérable. Leur but est multiple : quantitatif, qualitatif, voire même judiciaire (POLGE H, I972). 
Toutes ces radiographies sont prises dans un rayonnement polychromatique qui nécessite, à tous les niveaux (prises de vues, développement) de très grandes précautions pour obtenir un résultat valable.

I1 nous a semblé intéressant de faire ces mêmes manipulations en rayonnement monochromatique afin de voir si d'éventuelles améliorations pouvaient en découler.

\section{MÉTHODE EXPÉRIMENTALE}

\section{A. - Émission}

Les tubes les plus couramment employés en cristallographie utilisent le rayonnement du cuivre, du fer ou du molybdène suivant le rayonnement désiré. La longueur d'onde et les raies d'émission de ces différents éléments sont parfaitement connues et dès lors, il est possible de calculer le coefficient d'absorption massique du matériau à radiographier en vue de son analyse (International Tables for X-ray Cristallography).

Ainsi, pour le rayonnement du cuivre $\left(\mathrm{K}_{\alpha}=\mathrm{I}, 54 \AA\right.$ ) et pour un bois composé de 70 p. Ioo de matière cellulosique et de 30 p. roo de Lignine (POLGE, 1966), on appliquera la formule :

$$
\stackrel{\mu}{\rho}=\Sigma_{i} g_{i}\left(\frac{\mu}{\rho}\right) i
$$

où $g_{i}$ est la fraction de masse de l'élément $i$ dont le coefficient d'absorption massique est $\left(\frac{\mu}{\rho}\right) i$, $\Sigma$ symbolisant la sommation à l'ensemble des éléments $i$ du composant étudié.

\section{Exemple.}

Pour le rayonnement $K_{\alpha}$ du cuive (International Tables), on a pour $\frac{\mu}{p}$ :

$$
\begin{aligned}
& \mathrm{C}=4,60 \\
& \mathrm{H}=0,435 \\
& \mathrm{C}=\mathrm{I}, \mathbf{5 , 5}
\end{aligned}
$$

$$
\begin{gathered}
\text { D'où } \frac{\mu}{p} \text { cellulose }=8,1+\mathrm{cm}^{2} / \mathrm{g} \\
\text { et }{ }_{p}^{\mu} \text { lignine }-3,44 \mathrm{~cm}^{2} / g .
\end{gathered}
$$

Fn appliquant ces coefficients à l'absorption du bois, on obtient :

$$
\stackrel{\mu}{\rho}(\text { matière ligneuse })=\frac{70 \times 8,44+30 \times 3,44}{\mathrm{IOO}}=6,750 \mathrm{~cm}^{2} / \mathrm{g} .
$$

Ceci pour la matière ligneuse. La densité de la matière ligneuse est admise constamment égale à 1,53 (POLge H,).

Ainsi, pour un résineux de densité $0,5 \mathrm{I}$, on aboutit à un coefficient d'absorption massique de $2.24 \mathrm{~cm}^{2} / \mathrm{g}$ suivant la formule :

$$
\frac{\mu}{p} \times \frac{d}{\mathrm{I}, 53}
$$

Ce coefficient est une constante parfaitement stable liée de façon univoque à la longueur d'onde utilisée.

Pour exciter une raie caractéristique d'un élément par bombardement électronique, il faut donner aux électrons une énergie suffisante, au moins égale ou supéri eure à l'énergie du rayon- 
nement excité. Par exemple, pour le rayonnement du cuivre, la différence de potentiel sera égale :

$$
V_{0}=\frac{12372}{\lambda \mathrm{Cu}_{\alpha}}=\frac{1237^{2}}{\mathrm{I}, 54}=8 \text { ooo volts }
$$

Différents auteurs ont montré soit expérimentalement, soit théoriquement que le meilleur rapport d'intensité de la raie caractéristique, par rapport au fond continu émis est tel que $\frac{\mathrm{V}}{\mathrm{V}_{0}}=+$ (fig. I).

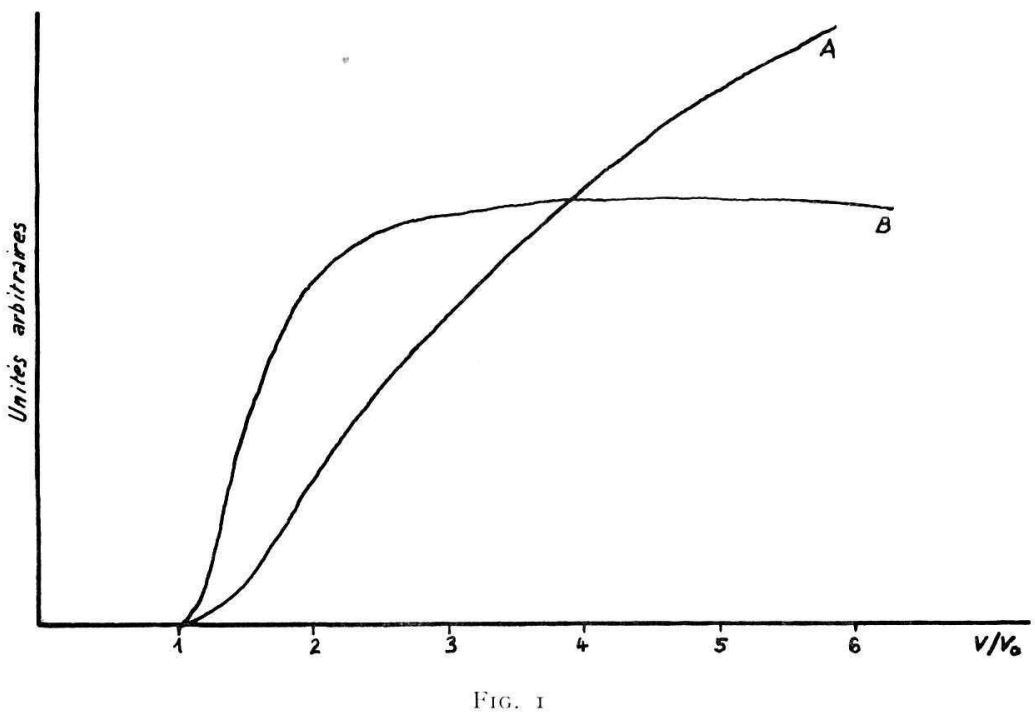

A : Intensité maximale d'une raie caractéristique $\mathrm{K}_{\alpha}$ en fonction de la tension de fonctionnement. Maximum intensity of a characteristic $\mathrm{K}_{\alpha}$ ray, versus the functioning tension (arbitrary units).

$\mathrm{B}$ : Rapport des intensités de la raie $\mathrm{K}_{\alpha}$ et du spectre continu. Ratio of the $\mathrm{K}_{x}$ ray intensity to the continuous spectrum intensity.

Donc, nous avons intérêt à travailler aux environ de $30 \mathrm{kV}$.

Le temps d'exposition ne dépendra que de l'intensité du faisceau d'électrons incidents. Il est bien entendu que nous travaillons en tension constante et continue de façon à maîtriser le profil du spectre d'émission.

Pour améliorer le rapport raie caractéristique/émission blanche, on interpose un filtre entre la source et l'échantillon, tel que sa raie d'absorption soit située juste à côté de la raie d'émission recherchée, vers les courtes longueurs d'ondes. Pour le Cuivre, on utilise un filtre de Nickel de 0,0 $5 \mathrm{~mm}$ d'épaisseur qui donne une perte de $45 \mathrm{p}$. Ioo sur la raie caractéristique. Mais il absorbe à $98 \mathrm{p}$. I oo la raie $\mathrm{K} \beta$ et il tronque le spectre blanc du côté des grandes longueurs d'onde de façon très efficace (fig. 2).

On obtient ainsi un rendement de $90 \mathrm{p}$. Ioo sur la raie monochromatique de l'élément de l'anticathode (GUINIER).

Le deuxième problème soulevé était celui de l'isodose. Les tubes générateurs de rayons X ont un foyer ponctuel de faible dimension. Les indicatrices d'isodose pour le fond blanc ne sont pas sphériques, elles présentent un maximum pour une incidence de l'anticathode de l'ordre de 30 p. 100 (Polge, I966).

Par contre, pour le rayonnement monochromatique de l'élément excité, l'indicatrice est pratiquement sphérique, avec un affaiblissement dû aux imperfections de surface pour les faisceaux émergeant tangentiellement au plan de l'anode (fig. 3).

Il suffit de placer un écran fluorescent pour déterminer où se situe le faisceau et avoir ses dimensions. 


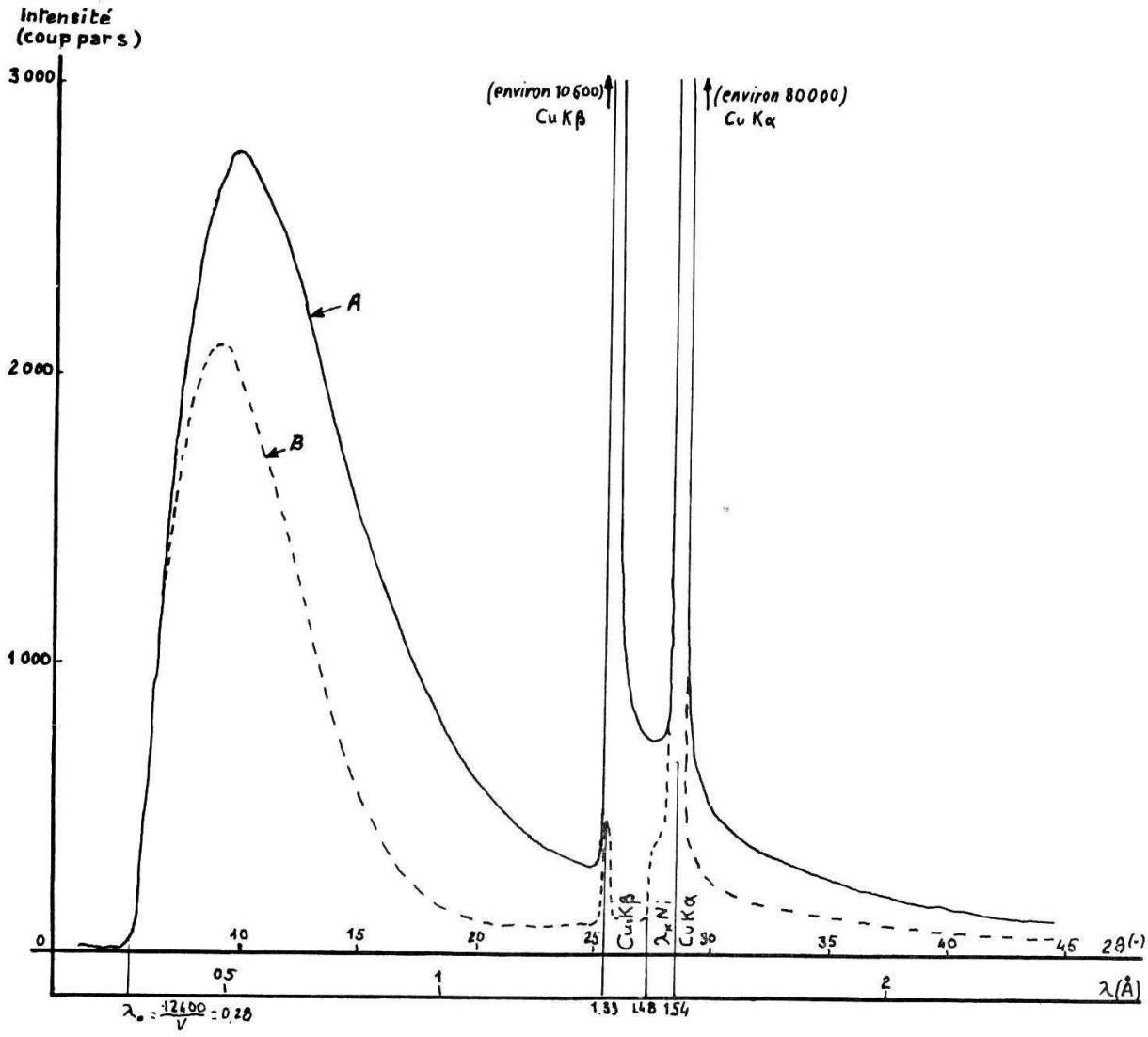

FIG. 2

A : Spectre du rayonnement d'un tube à anticathode de cuivre fonctionnant en courant redressé (potentiel de crête $40 \mathrm{kV}$ ). Spectromètre à crista! do Si (III). Récepteur : compteur à scintillation ( $\mathrm{Na} \mathrm{I}$ activité au thallium).

Radiation spectrum of a copper anticathode tube working with redressed current (peak potential $40 \mathrm{kV}$ ). Si-cristal spectrometer ( I I I). Receptor = scintillation counter (thallium-activated $\mathrm{Na} \mathrm{I).}$

I3: Spectre du même rayonnement aprés interposition d'un écran de $0,02 \mathrm{~mm}$ de nickel (d'après Parrish).

Spectrum of the same radiation, with a $0,0:$ mm nickel filter (from Parrish).

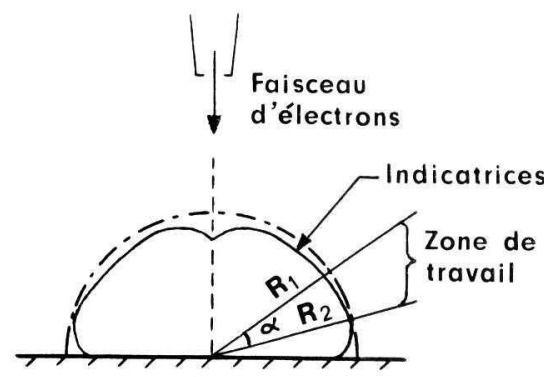

Anticathode

Rayonnement polychromatique $\left(\mathbf{R}_{1} \neq \mathbf{R}_{2}\right)$

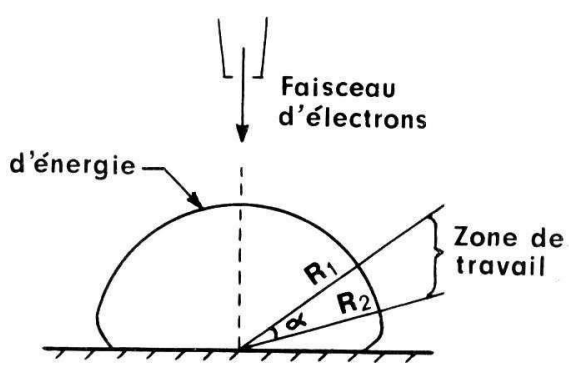

Anticathode

Rayonnement monochromatique

$$
\left(\mathbf{R}_{1}=\mathbf{R}_{2}\right)
$$

liti. 3. - Indicatrices d'énergie pour les différents ravonnements linergy indicators for the different radiations: 
Dans le cas qui nous intéresse (irradiation) d'un film $18 \times 24 \mathrm{~cm}$, à la distance de 3 mètres, on couvre un cercle isodosimétrique de $30 \mathrm{~cm}$ de diamètre (fig. 4).

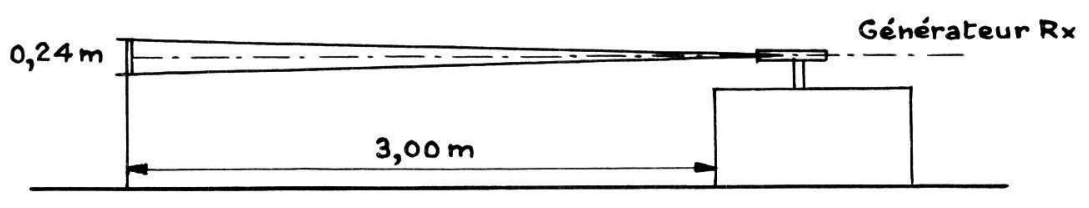

FIG. 4. - Irradiation horizontale

Horizontal irradiation

Il en résulte que la dissymétrie d'émission du fait de la cohérence de l'isodose est de l'ordre de $1 / 100$, ce qui est inférieur aux erreurs photométriques intrinsèques.

Le tube utilisé irradie dans le sens horizontal. Un petit montage a été nécessaire afin de placer les carottes verticalement (cf. fig. 5).

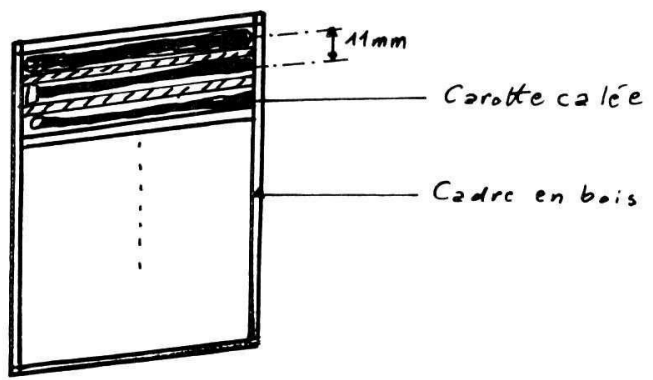

FIG. 5. - Cadre porte carotte

Wood-sample holder

On peut monter I 8 carottes simultanément sur un tel cadre.

Du fait de l'espacement rigoureux de chaque carotte (I I $\mathrm{mm}$ entre le milieu de chacune), l'analyse densitométrique est grandement facilitée.

En utilisant les formules décrites par PoLge (1966), ainsi que sa méthodologie, on arrive, à un temps de pose de 2 minutes avec $30 \mathrm{kV}$ et $4,5 \mathrm{mmA}$, à des contrastes satisfaisants pour l'analyse densitométrique.

$$
\text { B. Réception }
$$

11 restait à solutionner le problème du développement et de l'analyse des radios.

Une émulsion est caractérisée par le nombre de grains maximum développables par unité de surface, soit $\mathrm{N}_{m}$

$\mathrm{Ph}$, photons $\mathrm{X}$ d'énergie définie $\left(\gamma=I_{1,54} \AA\right)$ produisant $\mathrm{N}$ grains photographiques suivant la relation:

$$
\frac{\mathrm{N}}{\mathrm{N}_{m}}=\mathrm{I}-e^{-k \cdot \mathrm{Ph} .}
$$

$k$ étant une constante du film, qui dépend de sa section efficace et de sa fabrication.

Nous pouvons considérer que pour une émulsion traditionnelle (Definix, Kodirex etc.), on obtient la densité optique de I pour $\mathrm{S} \cdot \mathrm{IO}^{7}$ photons absorbés par $\mathrm{cm}^{2}$ (GUINIER).

Le film a reçu alors environ I photon par micron carré. D’après la loi de Beer, on a :

$$
k \cdot \log \frac{\mathrm{I}_{0}}{\mathrm{I}}=\mathrm{N}=\mathrm{D} \text { qui définit la densité optique, }
$$


$\mathrm{I}_{\mathbf{0}}=$ lumière incidente,

$\mathrm{I}=$ intensité reçue,

$\mathrm{N}=$ nombre de grain par unité de surface.

D'où :



où

$$
\frac{\mathrm{D}}{\mathrm{D}_{m}}=\frac{\mathrm{N}}{\mathrm{N}_{m}} \quad \therefore \mathrm{I}-e \text { k.Pl. }
$$

Cette relation dite de Eddler se calcule de façon rigoureuse et vérifie les lois de noircissement des films commerciaux RX à I ou 2 p. Ioo près pour l'ensemble des films (Morimoto).

La courbe de réponse du film étant parfaitement connue, il suffit alors de développer "à fond" pour que tous les grains soient révélés sans faire apparaître le bruit chimique. Il devient donc inutile de mettre au point des dispositifs plus ou moins compliqués de développenıent.

\section{Le problème du contraste}

La méthode monochromatique conduit à une variation totale de densité optique plus faible (si on utilise les mêmes coins optiques) que la méthode polychromatique. Mais, il ne faut pas perdre de vue que le but de la méthode est de réaliser une mesure quantitative de la variation de la densité du bois.

Il nous faut obtenir des radiographies à contraste moyen afin de ne pas atteindre une densité de noircissement trop grande, car, aux grandes densités optiques, toutes les lois quantitatives sont sujettes à erreur et par ailleurs correspondent à une faible sensibilité

Par surcroît, les microdensitomètres travaillent avec des faisceaux de lumière très faibles et les récepteurs de lumière (amplificateurs) restituent un bruit de fond électronique pour aboutir à un rapport signal-bruit tendant vers l'unité. Ce qui est non significatif et aboutit à une perte d'information (BRILLOIN).

A l'extrême, un cliché très contrasté se traduit par des plages blanches et noires (c'est-à-dire par o ou I). Toute dynamique de mesure est alors perdue. La seule chose que l'on peut alors dire est que le bois est moins dense (noir), plus dense (blanc) qu'une certaine densité, dite de seuil. Ce n'est donc pas le cliché le plus agréable à l'ceil qui donnera la meilleure information à l'enregistrement.

L'intérêt d'un contraste moyen est de se trouver dans la région où la loi de noircissement se vérifie à mieux que I p. Ioo et que la sensibilité $(\Delta D=$ différence de densité optique la plus petite mesurable) soit la plus petite.

Par exemple, avec un coin photographique explorant entre $\mathrm{D}=0,5$ et $\mathrm{D} \quad-\quad 1,5$, nous disposerons de 200 points de mesures (largeur de la feuille - longueur du coin) d'où un $\Delta \mathrm{D}$ de $\frac{1}{200}=0,005$.

Si nous disposons de clichés à contrastes trop élevés, pour la même longueur de coin et des densités variant entre $\mathrm{D}=0,5$ et $\mathrm{D}=3$.

$$
\Delta \mathrm{D}=\frac{2,5}{200}=0,0125
$$

ce qui est beaucoup moins sensible et se prètera moins bien au traitement numérique

Un autre intérêt est que pour des clichés à faible contraste, la courbe de noircissement théorique vérifie de façon très satisfaisante la courbe de noircissement expérimentale (Morimoto).

\section{RÉSULTATS}

Les radios de carottes de sondage ont donné des enregistrements densitométriques semblables à ceux obtenus pour ces mêmes carottes par la méthode PoLGE. Le fond était nettement décalé vers le bas, ce qui faisait que seul le signal "bois " était analysé (cf. fig. 6). 

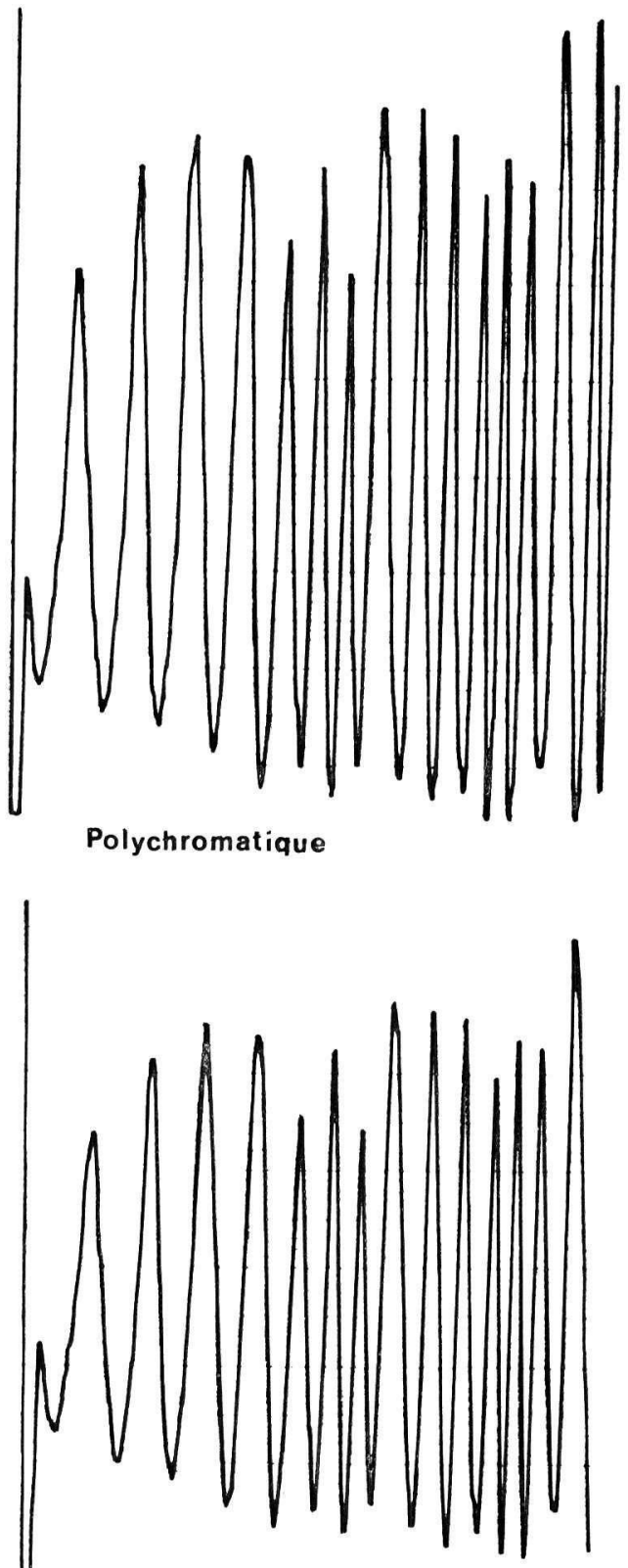

Monochromatique

Iı́. 6. - Enregistrements d'une même carotte de Pin sylvestre (échelle des abcisses : I/I) Recordings of a same boring sample of scots Pine (abscissa scale : I/I) 
Pour quantifier les résultats, on peut, soit faire appel à un coin de cellophane (cale à ro paliers par exemple) ou, plus simplement peser le matériau et, après calcul de la densité optique globale (planimètre ou microdensitomètre relié à un système d'enregistrement), relier directement la densité du matériau à la densité optique.

Outre la simplicité des manipulations, leur rapidité et l'éviction de différents problèmes délicats (isodose, développement, relation pseudolinéaire entre la densité du matériau et la densité optique), la méthode permet d'utiliser des radiographies effectuées dans n'importe quel laboratoire ou organisme possédant des tubes adéquats Minéralogie, Cristallographie, Science de la Terre etc.).

$$
\text { Reçu pour publication en octobre } 197.5 .
$$

\author{
SUMMARY \\ WOOD SAMPLE RADIOGRAPHY USING MONOCHROMATIC RAYS
}

Under the instigation of PoLge $\mathrm{H}$, the study of wood samples with $\mathrm{X}$ Rays has recently received a considerable impulse.

In this study we use moncchromatic radiation instead of polychromatic, which presents a few advantages.

The most used X Ray tube in crystallography employs the radiations of copper, iron or molytdenum, of which the wave-lengths and rays of emission are perfectly known.

Thus we are able to compute with a great precision the mass absorption factor of the analysed material and the tension which is applied to the terminals of the tube with regard to the wanted results. The contrast is obtained by changing the time of exposure.

Another particularity of monochromatic radiation is the coherence of the emission, which eliminates the problem of uniformity of allowed intensity.

Also, the perfect knowledge of the caracteristics of the different commercially available films lifts the problem of development, since then all the grains of the film are developed.

It is then easy, controlling all the factors of emission, absorption and development, to analyse the radiographs of wood samples in view of determining their density.

\title{
RÉFÉRENCES BIBLIOGRAPHIQUES
}

Brillorn L., 1972. La science et la théorie de l'Information. Dunod

Guinier A., I956. Théorie et technique de la radiocristallographie. (Dunod)

International Tables for X ray Crystallography. Vol. III, I962-2.2. et 3.2.(Kynoch Press, Birmingham).

Morimoto, ig63. A second comparison of various commercially available X-Ray Films. Acta Cryst., 16, IIO7-III9.

Polge H., 1966. Établissement des courbes de variation de la densité du bois. Ann. Sci. forest., 23 (I).

POLGE H., I972. Expertise judiciaire et fausses marques. Rev. forest. fr., mai-juin $1972, \mathrm{n}^{\circ} 3, \mathrm{I} 77^{-1} 86$. 